\title{
Human behavior, pathology and self-regulation
}

\author{
Comportamento humano, patologia e auto-regulação
}

Sam Goldstein?

At some point along the bell curve human behavior crosses a threshold of pathology and falls within the boundaries of clinical psychiatry. Yet psychiatric pathology is not a black and white phenomenon. Conditions of the DSM and ICD are not discrete entities. Many symptoms of psychiatric conditions represent qualities of human behavior distributed along a normal bell curve. Individuals at the lower tail of the curve are so different from others and experience such a severe level of adversity and impairment as to warrant diagnosis and intervention. In fact, it is increasingly apparent the bell curve phenomenon is reflective of many of the most common psychiatric conditions. As efforts are underway to create DSM-V, it is also increasingly apparent that a symptom count system is necessary but not sufficient to provide valid and reliable diagnostic categories. In facts, recent editions of the DSM and ICD have focused increasingly on age of onset, situational presentation and most importantly, levels of impairment. Relative to impairment, equal presentation of symptoms is not the equivalent of suggesting equal presentation of impairment (Gordon et al., 2006). In the condition currently defined in the DSM as attention deficit hyperactivity disorder, these researchers demonstrated that symptom presentation and severity predicts only a part of the variance in explaining overall level of impairment in every day life.

It is also increasingly recognized that weaknesses in certain neuropsychological processes such as self-regulation, executive functioning or planning contribute to psychiatric symptom presentation and impairment. These therefore represent the foundational explanations for certain psychiatric conditions. ADHD is one such condition in which symptoms and, to some extent, impairment are driven by weak abilities. Weaknesses in executive functions related to planning, working memory and impulse control compromise an individual's ability to meet daily expectations leading to symptoms and impairments crossing clinical, diagnostic thresholds (Boonstra et al., 2005).

Rates of ADHD among relatives of each subtype group of ADHD have been found to be greater than among relatives of controls (Faraone et al., 2000). The genetic contribution to ADHD has been postulated by a number of authors (Hechtman, 1993; Swanson et al., 2000). The underlying genetic mechanism has been suggested to be associated with multiple genes principally involving polymorphisms in the dopamine system. The heritability of ADHD has also repeatedly been found to be high. As Levy et al. (1997) note ADHD has an exceptionally high heritability compared with other behavioral disorders. These authors reported that $82 \%$ of monozygotic twins and $38 \%$ of dizygotic twins made an eight symptom ADHD cut-off for proband concordances.

1 George Mason University, USA.

Endereço para correspondência: Sam Goldstein 
In the last fifteen years, the biopsychosocial nature of ADHD across the life span has become increasingly apparent. Researchers have demonstrated that children, adolescents and adults with ADHD present with longer delay when their attention is misdirected, have difficulty switching when misdirected and on a battery of neuropsychological tasks perform poorly relative to others for visual tracking, auditory attention, application of intellectual skills and measures of continuous performance. Deficits on these tasks suggest problems with executive control likely linked to a deregulation of the frontal lobes of the brain (Castellenos, 1997; Pliszka et al., 2006). This pattern of problems, though not always the consensus reached by all researchers, has provided consistent evidence of deficits in a variety of tasks sensitive to executive functions and self-regulation in ADHD. As Barkley (1997) notes, pervasive impairments caused by ADHD are driven by a set of poor neuropsychological processes involving: difficulty fixing on and sustaining mental images; referencing the past in relation to present events; imagining hypothetical futures that might result from those events; establishing goals and plans of actions; avoiding reacting to stimuli likely to interfere with goal directed behavior; poor utilization of internal speech; efficient regulation of affect and motivation; and problems analyzing and synthesizing information.

The body of the literature attesting to the emotional, cognitive, vocational, academic, substance use and criminal impairments fueled by ADHD is also growing. It has been estimated from the available literature that approximately one third of adults with ADHD progress satisfactorily into their adult years. Another one third continue to experience some problems while the final third continues to experience and often develop significant problems (for review see Resnick and College, 2005; Barkley, 2006). By combining a number of outcome studies, it is reasonable to conclude that although compensatory strategies may reduce the impairment caused by these symptoms for some individuals, many continue to struggle. In fact, a large emerging body of research suggests that the symptoms of ADHD continue to plague most affected children as they transition into adulthood (Riccio et al., 2005).

Emerging research is also demonstrating that the symptoms that drive daily impairment for ADHD do in fact change into the adult years. Among the goals of DSM-V is to identify an appropriate set of symptoms for diagnosing ADHD in adulthood and create a diagnostic system capable of appropriately identifying adults with ADHD as well as assigning risk and estimating prognosis based on symptom presentation, severity and impairment. The current DSM-IV-TR symptom list for ADHD was developed with, and field tested, on children. Yet, as Canadian researchers Gabrielle Weiss and Lillian Hechtman suggested nearly fifteen years ago, the continuity of childhood conditions into adulthood is far from simple (Weiss and Hechtman, 1993). Symptoms may persist into adulthood but changes in adult expectation and life style as well as the capacity of human beings to develop compensatory strategies makes the presentation of the condition such as ADHD different in adulthood. Recently, Drs. Russell Barkley and Kevin Murphy reported their conceptualization of a set of diagnostic criteria for adult ADHD (Barkley and Murphy, 2006). Of the nine items they suggest statistically set adults with ADHD apart from children, three appear on the current DSM-IV-TR diagnostic protocol, and the others do not. These items fall broadly under an umbrella of executive function.

Recent reports from the U.S. Centers for Disease Control (CDC, 2005), Medco Health Solutions (Medco, 2005) and the Oregon Health and Sciences University Drug Class Review Program (McDonough and Peterson, 2005) reflect the increasing identification of those with ADHD and subsequent treatment. They also reflect a need for continued and thoughtful research in this area. Though these reports can be easily misused by those with agendas concerning the diagnosis and treatment of ADHD, as well as the mental health field overall, they offer a solid foundation for continued study and research on the marked adverse life time problems caused for those individuals meeting the diagnostic criteria for ADHD - individuals falling at the tail end of the bell curve for executive skills. It has yet to be demonstrated the manner in which specific symptoms, severity of symptoms, related comorbid problems and protective factors interact to affect impairment and outcome in ADHD. It is unclear whether those with the greatest impairments and struggles relative to $A D H D$ in adult life are more symptomatic for ADHD, experience greater comorbid conditions or simply have had the benefit of fewer protective factors in their lives leading to a resilient outcome. However, what is abundantly clear are the needs of these patients. I urge researchers and clinicians in the field of ADHD to address these issues, in particular attending to rates of diagnosis, effectiveness of treatments over time and an appreciation of the distinction between symptoms and impairments. Controversy in ADHD must not be reduced to opinion based debates about etiology, definition, evaluation and treatment but focus upon the importance of understanding human development and behavior. In the end it is our responsibility to help our colleagues and society in general appreciate and understand the limitations suffered by individuals with ADHD, a neurologically based but environmentally driven condition. Most importantly, our work must be about providing appropriate care and assistance to those in need throughout the life span. 


\section{REFERENCES}

Barkley R. Attention Deficit Hyperactivity Disorder. 3. ed. New York: Guilford; 2006

Barkley RA. The Nature of Self-Control. New York: Guilford; 1997.

Barkley RA, Murphy K. Identifying new symptoms for diagnosing ADHD in adults. The ADHD Report, 4: 7-11, 2006.

Boonstra A, Ooosterlaan J, Sergeant JA, Buitelaar JK. Executive functioning in adult ADHD: A meta-analytic review. Psychological Med, 35:1097-108, 2005.

Castellenos FX. Toward a pathophysiology of ADHD. Clinical Pediatrics, 36:381-93, 1997.

Faraone SV, Biederman J, Friedman D. Validity of DSM-IV subtypes of Attention-Deficit/Hyperactivity Disorder: A family study perspective. J Am Ac Child Adolesc Psychiat, 59:300-7, 2000.

Gordon M, Antshel K, Faraone S, Barkley R, Lewandowski L, Hudziak JJ et al. Symptoms versus impairment: The case for respecting DSM-IV's Criterion D. J Attent Dis, 9:465-75, 2006.

Hechtman L. Genetic and neurobiological aspects of attention deficit hyperactivity disorder: a review. J Psychiatr Neurosci, 9:193-201, 1993.

Levy F, Hay DA, McStephen M, Wood C, Waldman I. Attention-deficit hyperactivity disorder: A category or a continuum? Genetic analysis of a large-scale twin study. J Am Ac Child Adolesc Psychiatr, 36:737-44, 1997.

Pliszka SR, Glahn DC, Semrud-Clikeman M, Franklin C, Perez R, Xiong J. Neuroimaging of inhibitory control areas in children with ADHD who are treatment naive or in longterm treatment. Am J Psychiatr, 163:1052-60, 2006.

Resnick RJ, College RM. Attention deficit hyperactivity disorder in teens and adults: They don't outgrow it. J Clin Psycholog (special issue - ADHD in Adolescents and Adults), 61:529-33, 2005.

Riccio CA, Wolf M, Davis B, Romine C, George C, Lee D. Attention deficit hyperactivity disorder: Manifestation in adulthood. Arch Clin Neuropsycholog, 20:249-69, 2005.

Weiss G, Hechtman L. Hyperactive children grown up. 2. ed. New York: Guilford; 1993. 